\title{
FACIAL CHARACTERIZATIONS OF COMPLEX LINDENSTRAUSS SPACES
}

BY

\author{
A. J. ELLIS, T. S. S. R. K. RAO, A. K. ROY AND U. UTTERSRUD
}

\begin{abstract}
We characterize complex Banach spaces $A$ whose Banach dual spaces are $L^{1}(\mu)$ spaces in terms of $L$-ideals generated by certain extremal subsets of the closed unit ball $K$ of $A^{*}$. Our treatment covers the case of spaces $A$ containing constant functions and also spaces not containing constants. Separable spaces are characterized in terms of $w^{*}$-compact sets of extreme points of $K$, whereas the nonseparable spaces necessitate usage of the $w^{*}$-closed faces of $K$. Our results represent natural extensions of known characterizations of Choquet simplexes. We obtain also a characterization of complex Lindenstrauss spaces in terms of boundary annihilating measures, and this leads to a characterization of the closed subalgebras of $C_{\mathrm{C}}(X)$ which are complex Lindenstrauss spaces.
\end{abstract}

1. Introduction. Let $S$ be a compact convex subset of a locally convex Hausdorff space and let $A(S)$ denote the Banach space of all real-valued continuous affine functions on $S$ for the supremum norm. It is well known (see Alfsen [1]) that $S$ may be identified with the state-space $\left\{\varphi \in A(S)^{*}: \varphi \geqslant 0, \varphi(1)=1\right\}$ of $A(S)$ and that the unit ball of $A(S)^{*}$ may be identified with $\operatorname{co}(S \cup-S)$. $S$ is called a (Choquet) simplex if $A(S)^{*}$ is a vector lattice for the natural dual partial ordering. The set of extreme points of $S$ will be denoted by $\partial S$.

If $F$ is a face of $S$ then the complementary set $F^{\prime}$ consists of the union of all faces of $S$ which are disjoint from $F$; each $x$ in $S$ may be decomposed $x=\lambda y+$ $(1-\lambda) z$ with $0 \leqslant \lambda \leqslant 1, y \in F, z \in F^{\prime}$. If $F^{\prime}$ is itself a face of $S$ and if the constant $\lambda$ is uniquely determined by $x$, then $F$ is called a parallel face of $S$, and if, in addition, $y$ and $z$ are uniquely determined by $x(0<\lambda<1)$ we say that $F$ is a split face of $S$.

Ellis [9] showed that $S$ is a simplex if and only if every closed face of $S$ is split. This result may be rephrased to state that $S$ is a simplex if and only if $\operatorname{lin}_{\mathrm{R}} F$ (the real-linear hull of $F$ ) is an $L$-ideal in $A(S)^{*}$ whenever $F$ is a closed face of $S$ (see Alfsen and Effros [2, p. 161]). (A closed linear subspace $L$ of a Banach space $V$ is called an $L$-ideal if there exists a projection $P$ from $V$ onto $L$ such that for each $x \in V,\|x\|=\|P x\|+\|x-P x\|$.) For this result it is only necessary to assume the condition for each peak face $F$ of $S$ (see §3). Earlier, Rogalski [21, Théorème 37] had shown that if $S$ is metrizable then $S$ is a simplex if and only if $\overline{c o} E$ is a split face of $S$ (equivalently $\operatorname{lin}_{\mathrm{R}} \overline{c o} E$ is an $L$-ideal in $A(S)^{*}$ ) wherever $E$ is a $w^{*}$-compact subset of $\partial S$. Ellis and Roy [10, Theorem 1] have shown that Rogalski's result does not extend to the general nonmetrizable case.

Received by the editors October 7, 1980.

1980 Mathematics Subject Classification. Primary 46B99; Secondary 46A55, 46E15, 46E30, 46J10.

(C) 1981 American Mathematical Society 0002-9947/81/0000-0509/\$04.50 
The compact convex set $S$ is a simplex if and only if $A(S)^{*}$ is isometrically isomorphic to a real $L^{1}(\mu)$ space, i.e. if and only if $A(S)$ is a real Lindenstrauss space (see, for example, Semadeni [22]). In this paper we study the analogues of the results just mentioned to complex Banach spaces, that is, we characterize complex Lindenstrauss spaces (complex Banach spaces whose duals are isometrically isomorphic to complex $L^{1}(\mu)$ spaces) in terms of $L$-ideals generated by certain extremal subsets of the dual unit ball.

In the first part of $\$ 3$ we consider the most natural complex analogue of $A(S)$ spaces, namely the closed linear subspaces $A$ of $C_{\mathbf{C}}(X)$ containing constants and separating points of $X$, where $X$ is a compact Hausdorff space. In this situation the state-space $S=\left\{\varphi \in A^{*}: \varphi(1)=1=\|\varphi\|\right\}$ plays an important and natural role. We show that $A$ is a complex Lindenstrauss space if and only if $\operatorname{lin}_{\mathbf{C}} F$ (the complex linear hull of $F$ ) is an $L$-ideal in $A^{*}$ whenever $F$ is a closed (peak) face of $S$. This generalizes the result of Ellis [9]. At the end of $\$ 2$ we adapt the example of Ellis and Roy [10] to show that there exists a nonseparable space $A$ which is not a complex Lindenstrauss space but for which $\operatorname{lin}_{\mathbf{C}} \overline{c o} T E$ is a ( $w^{*}$-closed) $L$-ideal in $A^{*}$ whenever $E$ is a $w^{*}$-compact subset of the closed unit ball $K$ of $A^{*}$. The methods used for these results are generally adaptations of the methods for $A(S)$ spaces.

The situation for complex Banach spaces $A$ whose unit balls possess no extreme points is more complicated. In this case we use the machinery of complex Choquet theory (see Phelps [20]) and rely heavily on the work of Effros [8] and Lima's characterization [17, Theorem 5.8]; the Bishop-Phelps theorem [3] also plays a crucial role here, as it does in the real situation (see Ellis [9]). In the first part of $\$ 2$ we show that for separable complex Banach spaces the result of Rogalski mentioned above has a natural analogue: $A$ is a complex Lindenstrauss space if and only if $\operatorname{lin}_{\mathrm{C}} \overline{\text { co }} T E$ is an $L$-ideal in $A^{*}$ (where $T$ denotes the unit circle) whenever $E$ is a $w^{*}$-compact subset of $\partial K$. In $\S 3$ the main result (Theorem 3.3) of the paper shows that, in general, $A$ is a complex Lindenstrauss space if and only if $\operatorname{lin}_{\mathbf{C}} F$ is an $L$-ideal in $A^{*}$ whenever $F$ is a $w^{*}$-closed face of $K$. The proof of this result depends on the corresponding result for spaces $A$ containing the constant functions. We conclude $\$ 3$ by giving a characterization of complex Lindenstrauss spaces in terms of norm-closed faces of the dual ball, and also by applying our previous results to characterize real Lindenstrauss spaces.

In $\$ 4$ we extend a measure-theoretic characterization of complex Lindenstrauss spaces, due to Hirsberg and Lazar [14], to the case without constants. This enables us to characterize the closed subalgebras of $C_{\mathrm{C}}(X)$ which are complex Lindenstrauss spaces.

If $X$ is any compact Hausdorff space then by a measure on $X$ we will always mean a regular Borel measure on $X$. If $A$ is a subspace of $C_{\mathrm{C}}(X)$ we will write $A^{\perp}$ for the set of measures $\mu$ on $X$ such that $\int f d \mu=0$ for all $f$ in $A$,

The third author wishes to thank the U. K. Science Research Council for financial support during the writing of part of this paper. 
2. Separable spaces. In order to prove the characterization of separable complex Lindenstrauss spaces which is the analogue of Rogalski's characterization of metrizable simplexes, we need to establish some properties of complex Lindenstrauss spaces which are valid in the nonseparable case also. We first recall some notation of complex Choquet theory (see Phelps [20]).

Let $A$ be a complex Banach space and let $K$, with the $w^{*}$-topology, denote the closed unit ball of $A^{*}$. If $g$ belongs to $C_{\mathrm{C}}(K)$ (the complex-valued continuous functions on $K$ ) we define a $T$-homogeneous function hom $g$ in $C_{\mathrm{C}}(K)$ by

$$
(\operatorname{hom} g)(L)=\int_{T} \overline{t g}(t L) d t, \quad L \in K,
$$

where $T=\{z \in \mathbf{C}:|z|=1\}$ and the integration is with respect to Haar measure on $T$. For a complex Borel measure $\mu$ on $K$ we define another complex Borel measure hom $\mu$, with $\|$ hom $\mu\|\leqslant\| \mu \|$, by

$$
(\text { hom } \mu)(g)=\mu(\text { hom } g), \quad g \in C_{\mathbf{C}}(K) \text {. }
$$

For any complex Borel measure $\mu$ on $K$ we can write $d \mu=h d|\mu|$, where $h$ is a Borel function with modulus 1 , and we define a positive Borel measure $R \mu$ on $K$ by

$$
(R \mu)(g)=\int_{K} g(h(L) L) d|\mu|(L), \quad g \in C_{\mathrm{C}}(K) .
$$

Then we have $\|R \mu\|=\|\mu\|$, hom $R \mu=$ hom $\mu$, and if $\mu$ is a probability measure on $K$ with resultant $L \in K$ with $\|L\|=1$ we have $R($ hom $\mu)=\mu$ (see Phelps [20]).

Effros [8] showed that $A$ is a complex Lindenstrauss space if and only if hom $\mu_{1}=$ hom $\mu_{2}$ wherever $\mu_{1}, \mu_{2}$ are maximal probability measures on $K$ with common resultant $L \in K$.

Proposition 2.1. Let $A$ be a complex Lindenstrauss space (not necessarily separable) and let $K$ be the closed unit ball of $A^{*}$. Let $E$ be a compact subset of $\partial K$, let $F=\overline{\operatorname{co}} T E$ and let $J=\operatorname{lin}_{\mathbf{C}} F$. Then $J$ is a $w^{*}$-closed L-ideal in $A^{*}$ such that $J \cap K=F$.

Proof. Clearly $F$ is contained in $J \cap K$. In order to show that $F=J \cap K$ we must show that if $x \in J \cap K$ with $\|x\|=1$ then $x \in F$. But such an $x$ can be written $x=\alpha p$ for some $\alpha>0$ and $p \in F$, and so it will be sufficient to prove that $p /\|p\|$ belongs to $F$ whenever $p$ belongs to $F$ with $0<\|p\|<1$.

Let $\mu$ be a maximal measure on $K$, supported by $T E$, with resultant $r(\mu)=p$, and let $\omega_{p}=R($ hom $\mu)$. Recourse to the definitions shows that hom $\mu$ and $\omega_{p}$ are also supported by $T E$. Choose a maximal probability measure $\nu$ on $K$ with $r(\nu)=p /\|p\|$, and let $q \in \partial K$. Then if

$$
\theta=\|p\| \nu+(1-\|p\|)\left(\frac{1}{2} \varepsilon_{q}+\frac{1}{2} \varepsilon_{-q}\right),
$$

$\theta$ is maximal with $r(\theta)=p$ and so Effros' characterization of complex Lindenstrauss spaces shows that hom $\theta=\operatorname{hom} \mu$. However hom $\theta=\|p\|$ hom $\nu$ because $\left(\right.$ hom $\left.\varepsilon_{q}\right)(g)=($ hom $g)(q)=-($ hom $g)(-q)=-\left(\right.$ hom $\left.\varepsilon_{-q}\right)(g)$ for $g$ in 
$C_{\mathrm{C}}(K)$. Therefore we have

$$
\omega_{p}=R(\operatorname{hom} \theta)=R(\|p\| \text { hom } \nu)=\|p\| R(\text { hom } \nu)=\|p\| \nu,
$$

so that $\nu$ is supported by $T E$. Hence $p /\|p\|$ belongs to $F$ and, consequently, $F=J \cap K$.

The Krein-Šmulian theorem now shows that $J$ is a $w^{*}$-closed linear subspace of $A^{*}$. To show that $J$ is an $L$-ideal we need to show that $J \cap \operatorname{co}\left(J^{\prime}\right)=0$, where $J^{\prime}$ is the complementary cone of $J$ (see Alfsen and Effros [2, p. 110]). Suppose that

$$
0 \neq x=\alpha r_{1}+(1-\alpha) r_{2}, \quad x \in J, 0 \neq r_{j} \in J^{\prime}, 0<\alpha<1 .
$$

Since $r_{1}, r_{2}$ belong to $J^{\prime}$ we have

$$
\operatorname{face}\left(r_{j} /\left\|r_{j}\right\|\right) \cap J=\varnothing
$$

and so $r_{j} /\left\|r_{j}\right\|$ belongs to $J^{\prime}$ for $j=1,2$. Let $p=x /\|x\|$ and put $\beta=\alpha\left\|r_{1}\right\|+$ $(1-\alpha)\left\|r_{2}\right\|$. Then we have

$$
\begin{aligned}
\beta^{-1}\left(\alpha\left\|r_{1}\right\|\left(r_{1} /\left\|r_{1}\right\|\right)\right. & \left.+(1-\alpha)\left\|r_{2}\right\|\left(r_{2} /\left\|r_{2}\right\|\right)\right) \\
& =x / \beta=\|x\| / \beta p+(1-\|x\| / \beta) 0 .
\end{aligned}
$$

Choose a probability measure $\mu$ on $K$, supported by $T E$, with $r(\mu)=p$, and choose maximal probability measures $\mu_{j}$ on $K$, supported by $J^{\prime}$ with $r\left(\mu_{j}\right)=r_{j} /\left\|r_{j}\right\|$. (For the latter choice see Alfsen and Effros [2, p. 113, Lemma 4.3].) Using Effros' characterization of complex Lindenstrauss spaces we obtain

$$
\alpha\left\|r_{1}\right\| \text { hom } \mu_{1}+(1-\alpha)\left\|r_{2}\right\| \text { hom } \mu_{2}=\|x\| \text { hom } \mu,
$$

and since hom $\mu_{j}$ is supported by $T J^{\prime}=J^{\prime}$ and hom $\mu$ is supported by $J$, we must have hom $\mu=0$. But then $r($ hom $\mu)=r(\mu)=x=0$, giving the required contradiction.

TheOREM 2.2. Let $A$ be a separable complex Banach space and let $K$ be the closed unit ball of $A^{*}$. Then $A$ is a complex Lindenstrauss space if and only if $J=$ $\operatorname{lin}_{\mathrm{C}} \overline{\mathrm{co}} T E$ is an L-ideal in $A^{*}$ whenever $E$ is a compact subset of $\partial K$.

Proof. The necessity of the condition is shown by Proposition 2.1, so we assume the condition concerning $E$ to prove sufficiency.

If we can show that whenever $x \in K,\|x\|=1$, such that $P x=x$ or $P x=0$ for all $L$-projections $P$ on $A^{*}$ then $x$ belongs to $\partial K$, we will have verified all the conditions of Lima [17, Theorem 5.8(ii)] and it will follow that $A$ is a complex Lindenstrauss space. Fix such an $x$ and choose a maximal probability measure $\mu$ on $K$ with $r(\mu)=x$. We will show that $\mu$ is supported by a single point, and thus $x$ belongs to $\partial K$.

Whenever $E$ is a $w^{*}$-compact subset of $\partial K$ we will show that $J \cap K=\overline{\text { co }} T E$. Since $J$ is norm-closed and is generated by a $w^{*}$-compact convex set it is $w^{*}$-closed (see Dunford and Schwartz [6, V.5.9]), and so if $J \cap K$ properly contains $\overline{\text { co }} T E$ there is an extreme point $y$ of $J \cap K$ not belonging to $T E$. Because $J$ is an $L$-ideal $y$ belongs to $\partial K$, and $T E$ is contained in $J_{y}^{\prime}$, the complementary $L$-ideal to $\operatorname{lin}_{\mathbf{C}}\{y\}$. Let $p \in \overline{\text { co }} T E$ and let $\eta$ be a probability measure on $T E$ with $r(\eta)=p$. If $e$ is the $L$-projection with range $\operatorname{lin}_{\mathbf{C}}\{y\}$ then the function $q \leadsto v \circ e(q)(q \in K, v \in A)$ is 
$w^{*}$-Borel and satisfies the barycentric calculus (see Alfsen and Effros [2, p. 113]). Therefore

$$
v \circ e(p)=\int v \circ e(t z) d \eta(t z)=0
$$

for all $v$ in $A$ and, consequently, $p$ belongs to $J_{y}^{\prime}$. But then $y \in J$ is contained in $J_{y}^{\prime}$, which gives a contradiction.

If $E$ is a compact subset of $\partial K$ let $P_{E}$ denote the $L$-projection associated with $J$. The condition on $x$ implies that either $x \in J$ or $x \in J^{\prime}$, where $J^{\prime}$ is the $L$-ideal complementary to $J$. Write $\mu_{1}=\mu \mid T E$ and $\mu_{2}=\mu \mid(\partial K \backslash T E)(\partial K$ is a Borel set since $A$ is separable). If $\mu_{j} \neq 0$ let $y_{j} \in K$ be the resultant of $\mu_{j} /\left\|\mu_{j}\right\|$, and let $x_{j}=\left\|\mu_{j}\right\| y_{j}$; otherwise put $x_{j}=0$. Then we have $x=x_{1}+x_{2}$ and, since $\left\|x_{j}\right\|<$ $\left\|\mu_{j}\right\|$, we have $1=\|x\|=\left\|x_{1}\right\|+\left\|x_{2}\right\|$ and, moreover, $x_{1} \in \overline{c o} T E$ so that $x_{1} \in J$.

If $x \in J^{\prime}$, then $x=\left(I-P_{E}\right) x=\left(I-P_{E}\right) x_{2}$, and, since $\left\|\left(I-P_{E}\right) x_{2}\right\| \leqslant\left\|x_{2}\right\|$, we must have $x_{1}=0$, that is, $\mu(T E)=0$.

On the other hand, suppose that $x$ belongs to $J$. Then $x=P_{E} x=x_{1}+P_{E} x_{2}$ implies that $\left\|P_{E} x_{2}\right\|=\left\|x_{2}\right\|$, so that $x_{2}$ belongs to $J$ also. If $x_{2} \neq 0$ then, since $\mu$ is supported by $\partial K$, we must have $\mu\left(E^{\prime}\right) \neq 0$ for some compact subset $E^{\prime}$ of $\partial K \backslash T E$. If $y$ denotes the resultant of $\mu \mid E^{\prime} / \mu\left(E^{\prime}\right)$, and if $u=\mu\left(E^{\prime}\right) y$, we can write $x=x_{1}+$ $u+v$ for some $v$ in $K$, where $1=\left\|x_{1}\right\|+\|u\|+\|v\|$. This implies that $u$ belongs to $J$. Since $J \cap K=\overline{c o} T E$ there exists a maximal probability measure $\nu$ on $K$, supported by $T E$, with $r(\nu)=u$. Then if $\nu^{\prime}=\mu \mid E^{\prime}$ the measure $\nu-\nu^{\prime}$ is a boundary affine dependence on $K$ and, since $\operatorname{lin}_{\mathbf{C}} \overline{c o} T E^{\prime}$ is a $w^{*}$-closed $L$-ideal, $\left(\nu-\nu^{\prime}\right) \mid T E^{\prime}$ annihilates $A_{0}(K)$ (see Alfsen and Effros [2, p. 115, Theorem 4.5]). Consequently $\nu^{\prime}$ and, hence, $\nu$ belong to $A_{0}(K)^{\perp}$, and so $u=r(\nu)=0$. This contradiction shows that $x_{2}=0$ and that $\mu(\partial K \backslash T E)=0$.

Suppose now that (supp $\mu) \cap \partial K$ contains points $z_{1}, z_{2}$ with $T z_{1} \cap T z_{2}=\varnothing$. We can find disjoint open neighbourhoods of $T z_{1}$ and $T z_{2}$, and, hence, we can find a compact subset $E$ of $\partial K$ with $\mu(T E)>0$ and $\mu(\partial K \backslash T E)>0$. The previous reasoning shows that these inequalities holding simultaneously is impossible. Therefore $\mu$ is supported by $T z$ for some $z \in \partial K$. However, since $x=r(\mu)$ and $\|x\|=1$ it is evident that $\mu$ is supported by a singleton, as required.

The proof of Theorem 2.2 depends heavily on the metrizability of $\partial K$, or at least on the fact that maximal measures on $K$ are supported by $\partial K$. Ellis and Roy [10, Theorem 1] have shown that Rogalski's characterization of standard simplexes [21, Théorème 37] does not extend to the general nonstandard case. We now adapt this construction to show that the separability condition may not be dropped from Theorem 2.2.

Let $S$ be a compact convex set and let $A_{\mathrm{C}}(S)$ denote the Banach space of all continuous complex-valued affine functions on $S$, with the supremum norm.

THEOREM 2.3. There exists a complex Banach space $A$ with dual unit ball $K$ which has the following properties:

(i) $J=\operatorname{lin}_{\mathbf{C}} \operatorname{co} T E$ is an L-ideal in $A^{*}$ whenever $E$ is a compact subset of $\partial K$;

(ii) $A$ is not a complex Lindenstrauss space. 
Proof. Let $S$ be the compact convex set which is constructed in Ellis and Roy [10, Theorem 1] (denoted there by $K$ ). Then $S$ is not a simplex, the compact subsets of $\partial S$ are the finite sets $E$ and co $E$ is a split face of $S$. Let $A=A_{\mathrm{C}}(S)$.

The space $A$ is selfadjoint with state-space $S$, and since $S$ is not a simplex it follows that $A$ is not a complex Lindenstrauss space (see Hirsberg and Lazar [14]). The map $\varphi \rightsquigarrow \varphi / \varphi(1)$ is a $w^{*}$-continuous mapping from $\partial K$ onto $\partial S$, and hence if $E$ is a compact subset of $\partial K$ then $\operatorname{lin}_{\mathbf{C}} E=J$ is finite dimensional, say $J=$ $\operatorname{lin}_{\mathbf{C}}\left\{x_{1}, \ldots, x_{n}\right\}$ where $x_{j} \in \partial S$ for $1 \leqslant j \leqslant n$. Since $F=\operatorname{co}\left\{x_{1}, \ldots, x_{n}\right\}$ is a split face of $S$, and since $A$ is selfadjoint, $\operatorname{co}(F \cup-i F)$ is split in $Z=\operatorname{co}(S \cup-i S)$ and therefore $J$ is an $L$-ideal (see Hirsberg [13, Corollary 2.7]).

Theorem 2.3 also gives an example of a non-Lindenstrauss space $A$ with the property that whenever $E$ is a compact subset of $\partial K$ every continuous linear functional on $\operatorname{lin}_{\mathbf{C}} E$ has a norm-preserving extension in $A$ (compare the example in Alfsen [1, Proposition II.3.19]).

3. General spaces. We saw, in Theorem 2.3 , that the characterization of separable complex Lindenstrauss spaces given in Theorem 2.2 is not valid in the general nonseparable situation. We now seek a characterization of complex Lindenstrauss spaces, valid in the separable and nonseparable cases, which is analogous to the facial characterization of simplexes given by Ellis [9]. Our present characterization will be in terms of faces of the dual unit ball $K$.

A face $F$ of a compact convex set $S$ is called a ( $w^{*}$-closed) peak face for $A(S)$ if $F=f^{-1}(0)$ for some nonnegative function $f$ in $A(S)$. Briem [5] generalized the result of Ellis mentioned above by showing that $S$ is a simplex if and only if every peak face of $S$ is parallel; a simple proof of Briem's result can be obtained by a slight modification of the proof of Ellis and Roy [10, Theorem 2]. This result will be used in what follows.

Firstly we consider the situation for complex Banach subspaces of $C_{\mathbf{C}}(X)$ containing constants, where $X$ is a compact Hausdorff space. The required characterization in this case follows closely the characterization of real Lindenstrauss spaces of the type $A(S)$, and is much more straightforward than the general case.

THEOREM 3.1. Let $A$ be a closed linear subspace of $C_{\mathbf{C}}(X)$ containing constants and separating the points of $X$, where $X$ is a compact Hausdorff space. Let $S$ be the state-space of $A$ and let $K$ be the unit ball of $A^{*}$. Then $A$ is a complex Lindenstrauss space if $J=\operatorname{lin}_{\mathrm{C}} F$ is an L-ideal in $A^{*}$ such that $J \cap K=\overline{\operatorname{co}} T F$ whenever $F$ is a peak face of $S$ for $A(S)$.

Proof. To prove that $A$ is a complex Lindenstrauss space it will be sufficient, using the result of Hirsberg and Lazar [14, Theorem 2], to show that the set $Z=\operatorname{co}(S \cup-i S)$ is a simplex. By Briem's characterization of simplexes it will be sufficient to show that each $A(Z)$-peak face of $Z$ is parallel.

Let $H=\operatorname{co}(F \cup-i G)$ be an $A(Z)$-peak face of $Z$, so that $F$ and $G$ are $A(S)$-peak faces of $S$. We first show that $J \cap S=F$, where $J=\operatorname{lin}_{\mathrm{C}} F$. If $\mathfrak{p}$ belongs to $J \cap S$ then, by hypothesis,

$$
\mathfrak{p}=w^{*}-\lim _{\alpha}\left\{\sum_{k=1}^{m_{\alpha}} \lambda_{k}^{(\alpha)} t_{k}^{(\alpha)} y_{k}^{(\alpha)}\right\},
$$


where $0 \leqslant \lambda_{k}^{(\alpha)} \leqslant 1, \sum_{k=1}^{m_{\alpha}} \lambda_{k}^{(\alpha)}=1, t_{k}^{(\alpha)} \in T, y_{k}^{(\alpha)} \in F$. In particular we have

$$
\mathfrak{p}(1)=1=\lim _{\alpha} \sum_{k=1}^{m_{\alpha}} \lambda_{k}^{(\alpha)} t_{k}^{(\alpha)}
$$

and it follows that

$$
\mathfrak{p}=\lim _{\alpha} \sum_{k=1}^{m_{\alpha}} \lambda_{k}^{(\alpha)} y_{k}^{(\alpha)} \in F
$$

as required. Similarly we obtain $\left(\operatorname{lin}_{\mathrm{C}} G\right) \cap(-i S)=-i G$.

Since $J$ is an $L$-ideal and $J \cap S=F$, a result of Hirsberg [13] shows that $J \cap Z=\operatorname{co}(F \cup-i F)$ is a split face of $Z$. Now if $\mu$ belongs to $A(Z)^{\perp} \cap M(\partial Z)$ (where $M(\partial Z)$ denotes the real boundary measures on $Z$ ), then $\mu \mid \operatorname{co}(F \cup-i F)$ belongs to $A(\operatorname{co}(F \cup-i F))$, and because $F$ is a parallel face of $\operatorname{co}(F \cup-i F)$, it follows that $\mu(F)=0$ (see Hirsberg [12]). Similarly we can show that $\mu(-i G)=0$ and, hence, $\mu(H)=0$. Therefore $H$ is a parallel face of $Z$, so that $Z$ is a simplex.

The converse of Theorem 3.1 is also true. This will follow from a more general result (Theorem 3.3) below. Variations on the hypotheses of Theorem 3.1 may be given, and these are discussed in the following corollary. In particular it will follow that if $A$ is assumed to be selfadjoint in Theorem 3.1 then the hypothesis $J \cap K=\overline{c o} T F$ may be dropped.

COROLlaRY 3.2. The hypotheses on $F$ in Theorem 3.1 may be replaced by either of the following two conditions:

(i) $F$ is a split face of $\operatorname{co}(F \cup-i F)$ and $J$ is an L-ideal whenever $F$ is an $A(S)$-peak face of $S$;

(ii) $J$ is an L-ideal such that $J \cap K=\overline{c o} T F$ whenever $F$ is a peak face of $S$ for re $A$, i.e. $F=f^{-1}(0) \cap S$ for some $f \in \operatorname{re~} A, f>0$.

Proof. (i). A straightforward verification shows that $J \cap S=F$ and the proof proceeds as before.

(ii). As in the theorem we can prove that $J \cap S=F$ and $J \cap(-i S)=-i F$, and so $\operatorname{co}(F \cup-i F)$ is a split face of $Z$. Now, using the result of Briem [4, Theorem 3], it follows that $A$ is selfadjoint and $S$ is a simplex. Consequently $A$ is a complex Lindenstrauss space.

We now turn to the general case in which $A$ is a closed linear subspace of $C_{\mathrm{C}}(X)$ separating points of $X$, but not in general containing the constants. Again, let $K$ denote the unit ball of $A^{*}$ with the $w^{*}$-topology.

Let $\varphi$ denote the topological embedding of $X$ in $K$, where $\varphi(x)(f)=f(x)$ for each $x$ in $X$ and $f$ in $A$. A complex Borel measure $\mu$ on $X$ will be called a boundary measure for $A$, denoted $\mu \in M(\partial A)$, if $|\mu| \circ \varphi^{-1}$ is a maximal measure on $K$. A closed subset $E$ of $X$ of the form $\varphi^{-1}(T F)$, where $F$ is a closed face of $K$, will be called an $M$-set for $A$ if $\mu \in A^{\perp} \cap M(\partial A)$ implies $\mu \mid E \in A^{\perp}$. Note that if $1 \in A$ and if $F$ is a closed face of the state-space $S$ of $A$, then the set $E$ is an $M$-set for $A$ in the usual sense (see Hirsberg [13]). 
THEOREM 3.3. Let $A$ be a closed linear subspace of $C_{\mathbf{C}}(X)$ separating the points of $X$, and let $K$ be the unit ball of $A^{*}$. Then the following statements are equivalent.

(i) $A$ is a complex Lindenstrauss space.

(ii) Whenever $F$ is a closed face of $K$ the set $E=\varphi^{-1}(T F)$ is an $M$-set for $A$.

(iii) $J=\operatorname{lin}_{\mathbf{C}} F$ is an $L$-ideal in $A^{*}$ whenever $F$ is a $w^{*}$-closed face of $K$.

Proof. (i) $\Rightarrow$ (ii). If $E$ satisfies the conditions of (ii) and if $\mu \in A^{\perp} \cap M(\partial A)$ with $\|\mu\|=1$, we must show that $\mu \mid E \in A$. The measure $\nu=\mu \circ \varphi^{-1}$ is a boundary measure on $K$ with $r(\nu)=0$ and, consequently (see Effros [8, Lemma 4.2]), $R \nu$ is a maximal probability measure on $K$ with $r(R \nu)=0$. Since $A$ is a complex Lindenstrauss space we have $\operatorname{hom}(R \nu)=\operatorname{hom}\left(\varepsilon_{0}\right)=0$, and therefore $\operatorname{hom}(R \nu) \mid T \varphi(E)$ $=0$. Since $T \varphi(E)$ is $T$-invariant, it follows that $\operatorname{hom}\{R \nu \mid T \varphi(E)\}=0$ and, hence, $r(R \nu \mid T \varphi(E))=0$ so that $\int_{T \varphi(E)} f d(R \nu)=0$ for all $f$ in $A$.

Choose functions $\left\{g_{\alpha}\right\}$ in $C_{\mathbf{R}}(K), 0 \leqslant g_{\alpha} \leqslant 1$, such that $\left\{g_{\alpha}\right\}$ is pointwise decreasing to $\chi_{T \varphi(E)}$. We have, for all $f$ in $A$,

$$
\begin{aligned}
0 & =\int_{T \varphi(E)} f d(R \nu)=\lim _{\alpha} \int_{K} f g_{\alpha} d(R \nu) \\
& =\lim _{\alpha} \int_{X} f(h(x) \varphi(x)) g_{\alpha}(h(x) \varphi(x)) d|\mu|(x),
\end{aligned}
$$

where $d \mu=h d|\mu|$ is the polar decomposition for $\mu$. Now if $x \in E$ we have $h(x) \varphi(x) \in T \varphi(E)$, so that $g_{\alpha}(h(x) \varphi(x)) \rightarrow 1$. If $x$ is not in $E$ then $h(x) \varphi(x)$ is not in $T \varphi(E)$, and so $g_{\alpha}(h(x) \varphi(x)) \rightarrow 0$. Therefore (*) gives

$$
0=\int_{E} f(h(x) \varphi(x)) d|\mu|(x)=\int_{E} f d \mu, \quad \forall f \in A .
$$

Consequently $E$ is an $M$-set for $A$.

(ii) $\Rightarrow$ (iii). Let $F$ be a closed face of $K$, and let $E=\varphi^{-1}(T F)$. We define a linear mapping $e: A^{*} \rightarrow A^{*}$ by

$$
e(\rho)(a)=\int_{E} a d \mu, \quad a \in A,
$$

where $p \in A^{*}$ and $\mu \in M(\partial A)$ represents $p$. The fact that $E$ is an $M$-set implies that $e$ is well defined. If $p \in F$ then $p=r(\lambda)$ for some maximal measure $\lambda$ on $K$ supported by $F$, and so $\lambda \circ \varphi \in M(\partial A)$ represents $p$ and is supported by $E$. Consequently

$$
e(p)(a)=\int a d(\lambda \circ \varphi)=p(a), \quad a \in A,
$$

so that $e(p)=p$ and hence the range of $e$ contains $J=\operatorname{lin}_{\mathrm{C}} F$. We will show that $e$ is an $L$-projection on $A^{*}$ with range equal to $J$.

If $0 \neq p \in A^{*}$ we can find, by Hustad's theorem (see Phelps [20, Theorem 2.2]), a measure $\mu \in M(\partial A)$ with $\|\mu\|=1$ representing $p /\|p\|$. Therefore

$$
e(p /\|p\|)(a)=\int_{E} a d \mu, \quad a \in A,
$$


and so

$$
r(R(\mu \mid E))=e(p /\|p\|) \in \overline{\mathrm{co}} T F .
$$

Hence the range of $e$ is contained in $\mathbf{R}^{+} \overline{\text { co }} T F$. However since $F$ is a $w^{*}$-compact convex set it is easy to see that $G=\{\alpha x: 0 \leqslant \alpha \leqslant 1, x \in F\}$ is $w^{*}$-compact and convex and that $T F$ is contained in $G-G+i(G-G)$. Consequently $\overline{c o} T F$ is contained in $\operatorname{lin}_{\mathrm{C}} F$, and therefore $J$ is the range of $e$.

If $p$ belongs to $A$ let $\mu \in M(\partial A)$ represent $p$ with $\|\mu\|=\|p\|$. Then, for $a$ in $A$,

$$
\begin{aligned}
p(a) & =\int_{X} a d \mu=\int_{E} a d \mu+\int_{X \backslash E} a d \mu \\
& =e(p)(a)+(p-e(p))(a)=\int_{E} a d \mu+(p-e(p))(a)
\end{aligned}
$$

so that

$$
\|e(p)\|+\|p-e(p)\| \leqslant\|\mu|E\|+\| \mu| X \backslash E\|=\|p\| .
$$

It follows that $e$ is an $L$-projection.

(iii) $\Rightarrow$ (i). If $F$ is a $w^{*}$-closed face of $K$, and if $J=\operatorname{lin}_{\mathbf{C}} F$, then $J \cap K=\overline{\text { co }} T F$. In fact if $J \cap K$ properly contains co $T F$ then there is an extreme point $x$ of $J \cap K$ not belonging to TF. Since $J$ is an $L$-ideal, $x$ belongs to $\partial K$ so that $J_{x}=\operatorname{lin}_{\mathbf{C}}\{x\}$ is an $L$-ideal disjoint from $F$. But then $F$, and consequently $J$, is contained in the complementary $L$-ideal $J_{x}^{\prime}$, giving a contradiction. Now let $v_{0} \in A,\left\|v_{0}\right\|=1$, be such that $F=\left\{x \in K\right.$ : re $\left.v_{0}(x)=1\right\}$ is nonempty. By hypothesis $J=\operatorname{lin}_{\mathbf{C}} F$ is a $w^{*}$-closed $L$-ideal in $A^{*}$ and so, if $I=J_{\perp}, I$ is an $M$-ideal in $A$ such that $J=(A / I)^{*}$, where $A / I$ has the quotient norm. We have

$$
\left\|v_{0}+I\right\|=\sup \left\{\left|v_{0}(x)\right|: x \in J \cap K\right\}=1,
$$

and since $J \cap K=\overline{c o} T F$ it follows that $T F$ contains all extreme points of the closed unit ball of $J$, giving

$$
\|v+I\|=\sup \{|v(x)|: x \in J \cap K\}=\sup \{|v(x)|: x \in F\}
$$

for $v+I \in A / I$. Consequently $A / I$ can be naturally embedded as a closed linear subspace of $A_{\mathrm{C}}(F), F$ being the state space of $A / I$ and $v_{0}+I$ mapping into the constant function 1 .

If $G$ is a $w^{*}$-closed face of $F$ then $G$ is a $w^{*}$-closed face of $K$ and so, by hypothesis, $\operatorname{lin}_{\mathrm{C}} G$ is a $w^{*}$-closed $L$-ideal in $A^{*}$.

Evidently $\operatorname{lin}_{\mathrm{C}} G$ is a $w^{*}$-closed $L$-ideal in $J$.

Therefore $A / I$ is a complex Lindenstrauss space, by Theorem 3.1.

As in the proof of Theorem 2.2, to prove that $A$ is a complex Lindenstrauss space it suffices to show that if $p \in K$, with $\|p\|=1$, has the property that $e p=p$ or 0 whenever $e$ is an $L$-projection on $A^{*}$ then $p$ is an extreme point of $K$. Suppose that $\|p\|=1$ and $e p=p$ or 0 for all $L$-projections $e$ on $A^{*}$. By the Bishop-Phelps theorem [3], given $\varepsilon>0$, we can find a point $p_{0}$ with $\left\|p-p_{0}\right\|<\varepsilon$ such that $p_{0}$ belongs to a $w^{*}$-closed $A$-peak face $F$ of $K$ of the kind considered earlier in this proof. If $e^{\prime}$ denotes the $L$-projection of $A^{*}$ onto the $L$-ideal $J=\operatorname{lin}_{\mathbf{C}} F$ we have $e^{\prime} p=p$ or 0 , and because $\left\|e^{\prime} p-p_{0}\right\|=\left\|e^{\prime}\left(p-p_{0}\right)\right\|<\varepsilon$, we must have $e^{\prime} p=p$ so 
that $p$ belongs to $J$. If $e$ is any $L$-projection on $J$ then $e \circ e^{\prime}$ is an $L$-projection on $A^{*}$, so that $e \circ e^{\prime}(p)=p$ or 0 . Consequently $e p=p$ or 0 and, because $J$ is an $L$-space, Lima's theorem [17, Theorem 5.8] shows that $p$ belongs to $\partial(K \cap J)=\partial \overline{(\operatorname{co}} T F)$. Therefore $t_{0} p \in \partial F$, for some $t_{0}$ in $T$, so that $p$ is an extreme point of $K$ as required.

Combining Corollary 3.2 and the implication (i) $\Rightarrow$ (iii) of Theorem 3.3 we obtain the following characterization of complex Lindenstrauss spaces containing the constants.

COROllary 3.4. Let $A$ be a closed linear subspace of $C_{\mathbf{C}}(X)$ containing constants and separating the points of $X$, and let $S, K$ denote, respectively, the state-space of $A$ and the unit ball of $A^{*}$. Then $A$ is a complex Lindenstrauss space if and only if $J=\operatorname{lin}_{\mathrm{C}} F$ is an L-ideal in $A^{*}$ such that $J \cap K=\overline{\mathrm{co}} T F$ whenever $F$ is a peak face of $S$ for re $A$.

The equivalence (i) $\Leftrightarrow$ (iii) of Theorem 3.3 leads to the following characterization of complex Lindenstrauss spaces in terms of the norm-closed faces of the dual unit ball.

Corollary 3.5. Let $A$ be a complex Banach space, and let $K$ be the closed unit ball of $A^{*}$. Then $A$ is a complex Lindenstrauss space if and only if $\operatorname{lin}_{\mathrm{C}} F$ is an $L$-ideal in $A^{*}$ whenever $F$ is a norm-closed face of $K$.

Proof. Suppose that $A$ has the property stated concerning norm-closed faces of $K$. Then if $F$ is a $w^{*}$-closed face of $K, \operatorname{lin}_{\mathrm{C}} F$ is a norm-closed $L$-ideal in $A^{*}$ and, hence, a $w^{*}$-closed $L$-ideal in $A^{*}$. Therefore Theorem 3.3 shows that $A$ is a complex Lindenstrauss space.

Conversely, if $A$ is a complex Lindenstrauss space then, using standard arguments, we may assume that $A^{*}$ is isometrically isomorphic to a space $L_{\mathbf{C}}^{1}(\mu)$ such that $A^{* *}$ is isometrically isomorphic to $L_{\mathbf{C}}^{\infty}(\mu)$. Let $F$ be a proper norm-closed face of $K$. Then (see Alfsen and Effros [2, p. 104]) $F$ is contained in a maximal proper (norm-closed) face $G$ of $K$. As observed by Olsen [19] (see also Nielsen and Olsen [18]) $G$ must have the form

$$
G=\left\{f \psi: f \geqslant 0, \int f d \mu=1\right\},
$$

where $\psi \in L_{\mathbf{C}}^{\infty}(\mu)$ and $|\psi|=1$ a.e. $(\mu)$. [To see this separate $G$ from the open unit ball of $A^{*}$ by means of some $\eta \in L_{\mathbf{C}}^{\infty}(\mu)$, so that $G \subseteq\left\{f: \int f \eta d \mu=1, \int|f| d \mu=\right.$ 1\}. If

$$
H=\left\{\gamma \in L_{\mathbf{C}}^{\infty}(\mu):\|\gamma\| \leqslant 1, \int \gamma f d \mu=1 \forall f \in G\right\},
$$

then $H$ is a $w^{*}$-compact face of the unit ball of $L_{\mathbf{C}}^{\infty}(\mu)$ and hence contains an extreme point $\psi_{0}$ with $\left|\psi_{0}\right|=1$. If we put $\psi=\bar{\psi}_{0}$ then $G \subseteq\left\{f \psi: f>0, \int f d \mu=1\right\}$, and the maximality of $G$ implies that equality holds.] Without loss of generality we may assume that $\psi=1$, so that $F$ is a face of the simplex $G$, where

$$
G=\left\{f \in L_{\mathbf{R}}^{1}(\mu): f \geqslant 0, \int f d \mu=1\right\} .
$$


Now $\operatorname{lin}_{\mathbf{R}} F$ is an $L$-ideal in $L_{\mathbf{R}}^{1}(\mu)$ (see Alfsen and Effros [2, p. 161]) and, by consideration of the fact that $\left(\operatorname{lin}_{\mathbf{R}} F\right)^{\perp}$ is an $M$-summand in $L_{\mathbf{R}}^{\infty}(\mu)$, it is easy to show that $\operatorname{lin}_{\mathbf{R}} F$ has the form $\left\{f \chi_{E}: f \in L_{\mathbf{R}}^{1}(\mu)\right\}$ for some $\mu$-measurable set $E$. Hence $\operatorname{lin}_{\mathbf{C}} F$ has the form $\left\{f \chi_{E}: f \in L_{\mathbf{C}}^{1}(\mu)\right\}$. It now follows easily that $\operatorname{lin}_{\mathbf{C}} F$ is an $L$-ideal in $L_{\mathbf{C}}^{1}(\mu)$.

Several authors have studied the facial structure of the dual unit ball $K$ of a complex Lindenstrauss space. Olsen [19] showed that if $E$ is a $w^{*}$-compact subset of $\partial K$ such that $E \cap t E=\varnothing$ for $t \in T \backslash\{1\}$, then $\overline{c o} E$ is a face of $K$. Nielsen and Olsen [18] showed that if $F$ is a $w^{*}$-closed (norm-closed) face of $K$ then $\operatorname{lin}_{\mathbf{C}} F$ is a $w^{*}$-closed (norm-closed) $L$-ideal.

The facial structure of the dual unit ball of real Lindenstrauss spaces has been studied by several authors, for example Alfsen and Effros [2], Effros [7], Lau [15] and Lazar and Lindenstrauss [16]. Uttersrud [23] has given a characterization of real Lindenstrauss spaces in terms of decomposability of $L$-ideals in the dual space.

Using the results of the authors just mentioned, and making the obvious alterations in the proofs of Theorems 2.2 and 3.3, we can obtain the following facial characterizations of real Lindenstrauss spaces.

TheOREM 3.6. Let $A$ be a real Banach space and let $K$ be the unit ball of $A^{*}$. The following statements hold.

(i) If $A$ is separable then $A$ is a real Lindenstrauss space if and only if $J=$ $\operatorname{lin}_{\mathbf{R}} \overline{c o} E$ is an L-ideal in $A^{*}$ whenever $E$ is a $w^{*}$-compact subset of $\partial K$.

(ii) $A$ is a real Lindenstrauss space if and only if $J=\operatorname{lin}_{\mathbf{R}} F$ is an L-ideal in $A^{*}$ whenever $F$ is $a w^{*}$-closed face of $K$.

If $S$ is the set referred to in the proof of Theorem 2.3 then the space $A=A(S)$ shows that the separability condition may not be removed from Theorem 3.6(i).

4. Some further results and examples. When $A$ is a closed linear subspace of $C_{\mathrm{C}}(X)$, containing constants and separating points of $X$, Fuhr and Phelps [11, Theorem 4.4] and Hirsberg and Lazar [14, Theorem 2] showed that $A$ is a complex Lindenstrauss space if and only if $A^{\perp} \cap M(\partial A)=\{0\}$. We give the analogue of this result in the case when $A$ does not contain the constant functions.

We will require the following notation used by Phelps [20]. If $A$ is a closed linear subspace of $C_{\mathrm{C}}(X)$ separating the points of $X$, we denote by $\tilde{A}$ the closed linear subspace of $C_{\mathrm{C}}(X)$ consisting of the functions $g$ in $C_{\mathrm{C}}(X)$ such that $\operatorname{sg}(x)=\operatorname{tg}(y)$ wherever $s, t \in I, x, y \in X$ and $s f(x)=t f(y)$ for all $f$ in $A$. If $\lambda$ and $\mu$ are complex measures on $X$ we write $\lambda \approx \mu$ if $\lambda(g)=\mu(g)$ for all $g$ in $\tilde{A}$. We retain the notation $\varphi$ from the previous section.

There exists (see Phelps [20]) a Borel measurable map $s: T \varphi(x) \rightarrow T \times X$ such that $s(L)=\left(t_{L}, x_{L}\right)$ and $L=t_{L} \varphi\left(x_{L}\right)$. Given a maximal probability measure $\mu$ on $K$ we define a complex boundary measure $H \mu$ where, for $g \in C_{\mathrm{C}}(X)$,

$$
\int g d H \mu=\int_{T_{\varphi(X)}} t_{L} g\left(x_{L}\right) d \mu(L) .
$$

If $\mathfrak{p}$ is the resultant of $\mu$ then $H \mu$ represents $\mathfrak{p}$. 
THEOREM 4.1. Let $A$ be a closed linear subspace of $C_{\mathrm{C}}(X)$, separating the points of $X$. Then the following statements are equivalent:

(i) $A$ is a complex Lindenstrauss space;

(ii) $\mu \in A^{\perp} \cap M(\partial A)$ implies $\mu \approx 0$.

Proof. (i) $\Rightarrow$ (ii). Let $\mu$ belong to $A^{\perp} \cap M(\partial A)$ with $\|\mu\|=1$. If $\nu=\mu \circ \varphi^{-1}$ then $R \nu$ is a maximal probability measure on $K$ with $r(R \nu)=0$, where $K$ is the closed unit ball of $A^{*}$ with the $w^{*}$-topology. Since $A$ is a complex Lindenstrauss space, hom $R \nu=0$ (Effros [8]), and so hom $\nu=$ hom $R \nu=0$. Therefore we have $\mu \approx 0$ (see Phelps [20, Proposition 3.5]).

(ii) $\Rightarrow$ (i). It will be sufficient to prove that if $\mu$ is a maximal probability measure on $K$ with $r(\mu)=0$ then hom $\mu=0$ (see Effros [8], in particular the proof of Theorem 4.3). If we write $\lambda=H \mu$ then $\lambda$ belongs to $A^{\perp} \cap M(\partial A)$, and so $\lambda \approx 0$. Therefore $\operatorname{hom}\left(H \mu \circ \varphi^{-1}\right)=0$ so that $\left(H \mu \circ \varphi^{-1}\right)(g)=0$ for all $T$-homogeneous continuous functions $g$ on $K$. For such functions $g$ we have

$$
\begin{aligned}
0 & =\left(H \mu \circ \varphi^{-1}\right)(g)=H \mu(g \circ \varphi)=\int_{T \varphi(X)} t_{L} g\left(\varphi\left(x_{L}\right)\right) d \mu \\
& =\int_{T \varphi(X)} g\left(t_{L} \varphi\left(x_{L}\right)\right) d \mu=\int g d \mu .
\end{aligned}
$$

Consequently hom $\mu=0$ as required.

The following result generalizes the result of Hirsberg and Lazar [14, Corollary 3.5].

Corollary 4.2. Let $A$ be a closed subalgebra of $C_{\mathrm{C}}(X)$, separating the points of $X$. Then $A$ is a complex Lindenstrauss space if and only if either $A=C_{\mathrm{C}}(X)$ or $A=\left\{f \in C_{\mathrm{C}}(X): f\left(x_{0}\right)=0\right\}$ for some $x_{0} \in X$.

Proof. We need only prove the necessity of the conclusion. Let $\nu$ be a measure on $X$ with $\nu \in A^{\perp}$. Then we can write $\nu=\alpha_{1} \nu_{1}-\alpha_{2} \nu_{2}+i\left(\alpha_{3} \nu_{3}-\alpha_{4} \nu_{4}\right)$, where $\alpha_{j} \geqslant 0$ and $\nu_{j}$ are probability measures on $X$. We can find probability boundary measures $\mu_{j}$ on $X$ such that $\mu_{j}-\nu_{j} \in A^{\perp}, j=1,2,3,4$, and so $\mu=\alpha_{1} \mu_{1}-\alpha_{2} \mu_{2}$ $+i\left(\alpha_{3} \mu_{3}-\alpha_{4} \mu_{4}\right)$ belongs to $A^{\perp} \cap M(\partial A)$. But then, by Theorem 4.1(ii), $\mu \approx 0$ and this implies that $\mu=0$ (see Phelps [20, Proposition 3.4]). But then $\alpha_{1} \mu_{1}-\alpha_{2} \mu_{2}$ $=0$ so that re $\nu \in A^{\perp}$. Consequently $A$ is selfadjoint and the conclusion follows from the Stone-Weierstrass theorem.

That the second possibility may occur in the above corollary is seen by considering the complex sequence space $c_{0}$.

In Theorem 3.1 the condition that $\operatorname{lin}_{\mathrm{C}} F$ is an $L$-ideal cannot be replaced by the condition that $\operatorname{lin}_{\mathbf{C}} F$ is a $w^{*}$-closed $L^{1}$-space; for example let $S$ be a square in $\mathbf{R}^{2}$ and let $A=A_{\mathrm{C}}(S)$. In the same theorem it is not sufficient to assume the conditions concerning $F$ for $A$-peak faces of $S$ only; in fact if $A$ is the disc algebra then the $A$-peak faces of $S$ are generated by the closed subsets of the unit circle with linear Lebesgue measure zero, and the complex-linear spans of such faces $F$ are $w^{*}$-closed $L$-ideals $J$ satisfying $J \cap K=\overline{\text { co }} T F$. 
The condition $J \cap K=\overline{c o} T F$ is not generally true for $w^{*}$-closed faces $F$ of the dual ball of a complex Banach space $A$, even if $J$ is a $w^{*}$-closed $L$-ideal in $A^{*}$. For example let $A=\mathrm{C}^{2}$ and let the norm in $A^{*}$ be given by

$$
\|(x, y)\|=\max \{|x|,|y|,|x+y|\} \text {. }
$$

Then if $F=\{(x, y): 0 \leqslant x, y, x+y=1\}, F$ is a closed face of $K$ such that $J=\operatorname{lin}_{\mathbf{C}} F=A^{*}$; however it can easily be checked that co $T F$ is properly contained in $K$-for example $(1,-1) \in K \backslash \overline{\text { co }} T F$.

Finally, the condition in Theorem 3.3(ii) that $\varphi^{-1}(T F)$ is an $M$-set whenever $F$ is a $w^{*}$-closed face of $K$ may not be replaced by the condition that $\varphi^{-1}(F)$ is an $M$-set. For example, let $A$ be the complex $G$-space $\left\{f \in C_{\mathbf{C}}[0,1]: f(0)=i f(1)\right\}$. Then $F=\varphi(0)$ is a $w^{*}$-closed face of $K$ such that $\{0\}=\varphi^{-1}(F)$ is not an $M$-set, whereas $\varphi^{-1}(T F)=\{0,1\}$ is an $M$-set for $A$.

\section{REFERENCES}

1. E. M. Alfsen, Compact convex sets and boundary integrals, Springer-Verlag, Berlin, 1971.

2. E. M. Alfsen and E. G. Effros, Structure in real Banach spaces. I, II, Ann. of Math. (2) 96 (1972), 98-173.

3. E. Bishop and R. R. Phelps, The support functionals of a convex set, Convexity, Proc. Sympos. Pure Math., vol. 7, Amer. Math. Soc., Providence, R. I., 1963.

4. E. Briem, Peak sets for the real part of a function algebra, Science Inst., Univ. of Iceland, Preprint $7,1977$.

5. A characterization of simplexes by parallel faces, Bull. London Math. Soc. 12 (1980), 55-59.

6. N. Dunford and J. T. Schwartz, Linear operators, Part I: General theory, Interscience, New York and London, 1958.

7. E. G. Effros, On a class of real Banach spaces, Israel J. Math. 9 (1971), 430-458.

8. On a class of complex Banach spaces, Illinois J. Math. 18 (1974), 48-59.

9. A. J. Ellis, A facial characterization of Choquet simplexes, Bull. London Math. Soc. 9 (1977), 326-327.

10. A. J. Ellis and A. K. Roy, Dilated sets and characterizations of simplexes, Invent. Math. 56 (1980), 101-108.

11. R. Fuhr and R. R. Phelps, Uniqueness of complex representing measures on the Choquet boundary, J. Funct. Anal. 14 (1973), 1-27.

12. B. Hirsberg, $A$ measure theoretic characterization of parallel and split faces and their connections with function spaces and algebras, Aarhus Univ. Various Publ. Ser. No. 16 (1970).

13. __ M-ideals in complex function spaces and algebras, Israel J. Math. 12 (1972), 133-146.

14. B. Hirsberg and A. J. Lazar, Complex Lindenstrauss spaces with extreme points, Trans. Amer. Math. Soc. 186 (1973), 141-150.

15. K. S. Lau, The dual ball of a Lindenstrauss space, Math. Scand. 33 (1973), 323-337.

16. A. J. Lazar and J. Lindenstrauss, Banach spaces whose duals are $L_{1}$ spaces and their representing matrices, Acta Math. 126 (1971), 165-193.

17. A. Lima, Intersection properties of balls and subspaces in Banach spaces, Trans. Amer. Math. Soc. 227 (1977), 1-62.

18. N. J. Nielsen and G. H. Olsen, Complex preduals of $L_{1}$ and subspaces of $l_{\infty}^{n}(C)$, Math. Scand. 40 (1977), 271-287.

19. G. H. Olsen, On the classification of complex Lindenstrauss spaces, Math. Scand. 35 (1974), 237-258.

20. R. R. Phelps, The Choquet representation in the complex case, Bull. Amer. Math. Soc. 83 (1977), 299-312.

21. M. Rogalski, Caractérisation des simplexes par des propriétés portant sur les faces fermées et sur les ensembles compacts de points extrémaux, Math. Scand. 28 (1971), 159-181. 
22. Z. Semadeni, Free compact convex sets, Bull. Acad. Polon. Sci. Sér. Sci. Math. 13 (1965), 141-146.

23. U. Uttersrud, On M-ideals and the Alfsen-Effros structure topology, Math. Scand. 43 (1978), 369-381.

Department of Pure Mathematics, The University College of Swansea, Swansea Sa2 8PP, WALES

Mathematical Division, Indian Statistical Institute, Calcutta 70035, India (Current address of T. S. S. R. K. Rao and A. K. Roy)

Telemark Regional College, 3800 BO, Norway (Current address of U. Uttersrud)

Current address (A. J. Ellis): Department of Mathematics, University of Hong Kong, Hong Kong 